\title{
Tuberculous Coxitis: Diagnostic Problems and Varieties of Treatment: A Case Report ${ }^{\S}$
}

\author{
H. Klein ${ }^{*}, 1$ J.B. Seeger ${ }^{1}$ and I. Schleicher ${ }^{2}$ \\ ${ }^{I}$ Department of Orthopaedics and Orthopaedic Surgery, University Hospital Giessen and Marburg (UKGM), \\ Klinikstraße 33, D-35392 Giessen, Germany \\ ${ }^{2}$ Department of Trauma Surgery, University of Giessen and Marburg, Klinikstraße 33, 35385 Giessen, Germany
}

\begin{abstract}
Although the prevalence of tuberculosis reduces, it still belongs to the most important infectious diseases worldwide even in industrial countries.

We report an unusual case of tuberculous coxitis in a 28 -year-old healthy native female with recurrent hip pain. While Xray and microbiological examination of the aspirate showed no abnormality, only extended diagnostic measurements and detailed history led to the diagnosis of TBC. Although the patient did not show any pulmonary symptoms open tuberculosis was confirmed. After a course of antibiotic treatment she underwent reconstructive surgery which consecutively improved range of motion. This case report emphasizes that tuberculosis should still be considered as a significant disease even in healthy patients with uncertain complaints in joints without significant initial radiographic abnormalities. We recommend the described diagnostic procedures as well as an antibiotic and surgical treatment.
\end{abstract}

Keywords: Tuberculous coxitis, TBC, tuberculosis.

\section{INTRODUCTION}

Although the prevalence constantly reduces [1] tuberculosis still belongs to the most important infectious diseases worldwide. The World Health Organisation (WHO) estimated the morbidity of 1.7 billion people in the year 1990, about more than 3 million people are still dying as a result of its affection every year $[2,3]$.

Analysis of 26.302 tuberculosis cases of a national german survey during the period from 1996-2000 showed a crude proportion of tuberculosis patients with extrapulmonary manifestation of $21.6 \%$, that was most likely among females, children aged under 15 years and immigrants from Asia and Africa [4]. In case of tuberculous coxitis (TBC) a prior pulmonary infection causes the affection of the joint by haematogenous spread. Early diagnosis can be difficult as the primary clinical symptoms at an early stage as well as radiological findings are often non-specific [5]. In addition to MRI and CT, diagnosis may be confirmed by joint aspiration and bacteriological culture, which unfortunately is negative in $30 \%$ of the cases [6], so consequently an open biopsy has to be performed.

We report an unusual case of TBC in a 28-year-old otherwise healthy native female with recurrent hip pain without a history of long-termed stays in foreign countries.

*Address correspondence to this author at the Department of Orthopaedics and Orthopaedic Surgery, University Hospital Giessen and Marburg (UKGM), Klinikstraße 33, D-35392 Giessen, Germany; Tel: +49-641-98556094; Fax: +49-641-985-42999;

E-mail: heiko.klein@ortho.med.uni-giessen.de

${ }^{\S}$ The case report was carried out at the Department of Orthopaedic Surgery, University Hospital Giessen and Marburg in Giessen, Germany.

\section{CASE REPORT}

A 28-year old native female patient complained about recurrent pain in the right hip joint without previous trauma progressing by physical strain starting about 1.5 years before. A MRI of the pelvis performed 3 months before admittance showed a swelling of the capsule and reduction of the cartilage in the right hip joint which was considered as an early arthritis or coxitis. After treatment with physiotherapy and oral non-steroidal antirheumatic medication the symptoms were regressive and no further diagnostic procedures were performed.

Caused by recently aggravation of pain in the right hip joint within the days before, the patient was not able to stand and walk and the range of motion (ROM) was painfully reduced. Blood samples were normal except slightly increased CRP of $4.2 \mathrm{mg} / 1$ (reference: $<0.5 \mathrm{mg} / \mathrm{l}$ ) without any local signs of inflammation in the pelvic region. X-ray of the right pelvis showed a slight joint space narrowing with affection of the acetabulum and the femoral head (Fig. 1). An effusion was detected by ultrasound, aspiration of the hip joint led to no further diagnosis as aerobic and anaerobic cultures were sterile. The MRI showed a joint space narrowing in the cranial part of the acetabulum with increased subchondral sclerosis as well as a synovial enhancement with bone marrow edema in the femoral head and the corresponding acetabulum.

The further clinical examination with detailed reconstruction of patient's history revealed a surgical procedure with extirpation of a lymph node from the left supraclavicular region two years before. The histological result was suspicious for tuberculosis with marked 


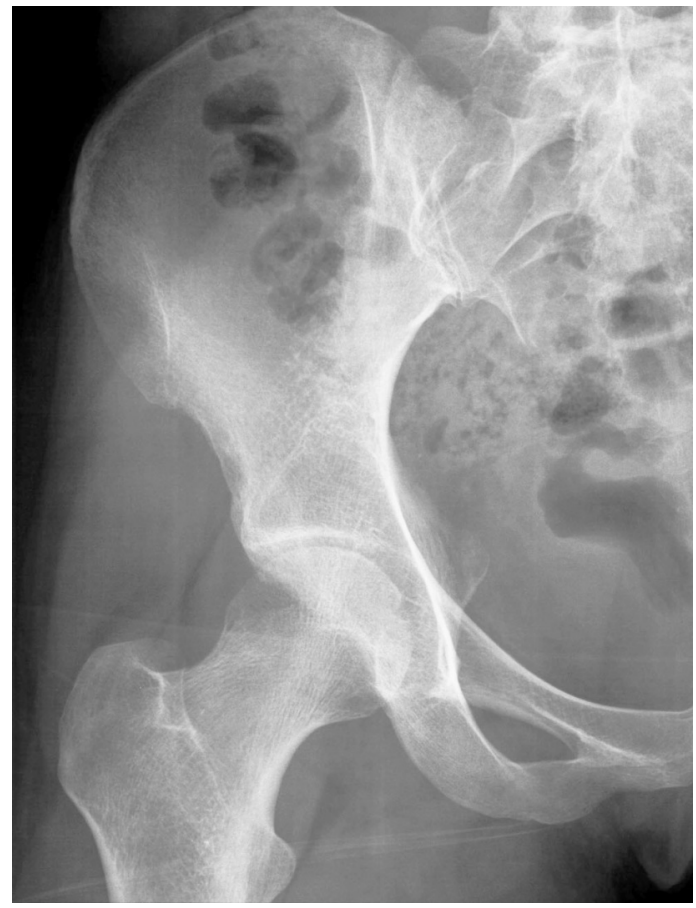

Fig. (1). Initial X-ray of the right pelvis showing only a slight joint space narrowing with affection of the acetabulum and the femoral head.

granulomatous lymphadenitis and caseous degenerated confluent necrosis, but tuberculin test and sputum sample examinations by microscopy and culture medium were negative. A CT without any proof of lymphadenopathy and pulmonary infiltration could not confirm this suspicion. In addition there were no other risk factors except her profession as a veterinarian, especially as she was neither an immigrant nor had long-termed stays in foreign countries. So no further treatment had been started at that time.

Although the initial microbiological examination of the first aspirate being inconspicuous, a current QuantiferonTest [7] showed a positive reaction as an indication of a latent or active infection with Mycobacterium tuberculosis (M. tbc), Mycobacterium Kansasii, Mycobacterium Szulgai or Mycobacterium Marinum. X-ray of the chest showed an enhanced parenchymal change in both apical segments. A consequently performed CT of the chest and abdomen revealed the clinical sign of reactivated tuberculosis in the apical and posterior bronchopulmonary segments with nodular peribronchiovascular focus as a sign of open lung tuberculosis. Furthermore a second aspiration of the right hip joint confirmed $M$. tbc by microscopical analysis and bacterial culture. Gastric secretion examined by polymerase chain reaction (PCR) as well as bacterial culture and microscopy of the sputum showed an amount of M. tbc.

Thereafter by confirming TBC and open lung tuberculosis, the patient was treated with combination of oral Isoniazid, Rifampicin, Ethambutol and Pyrazinamid. She received physiotherapy with partial weight bearing of $10 \mathrm{~kg}$.

Three months later X-ray showed an increasing patchy transparency in the femoral head and neck as well as in the greater trochanter and acetabulum with clear progressive joint space narrowing in the corresponding parts of the hip joint (Fig. 2). A complementary MRI with contrast medium five months after starting therapy showed active arthritis with progressive destruction of the right hip joint and a new appearance of liquid retention in the right acetabulum as a sign of tuberculous osteomyelitis (Fig. 3).

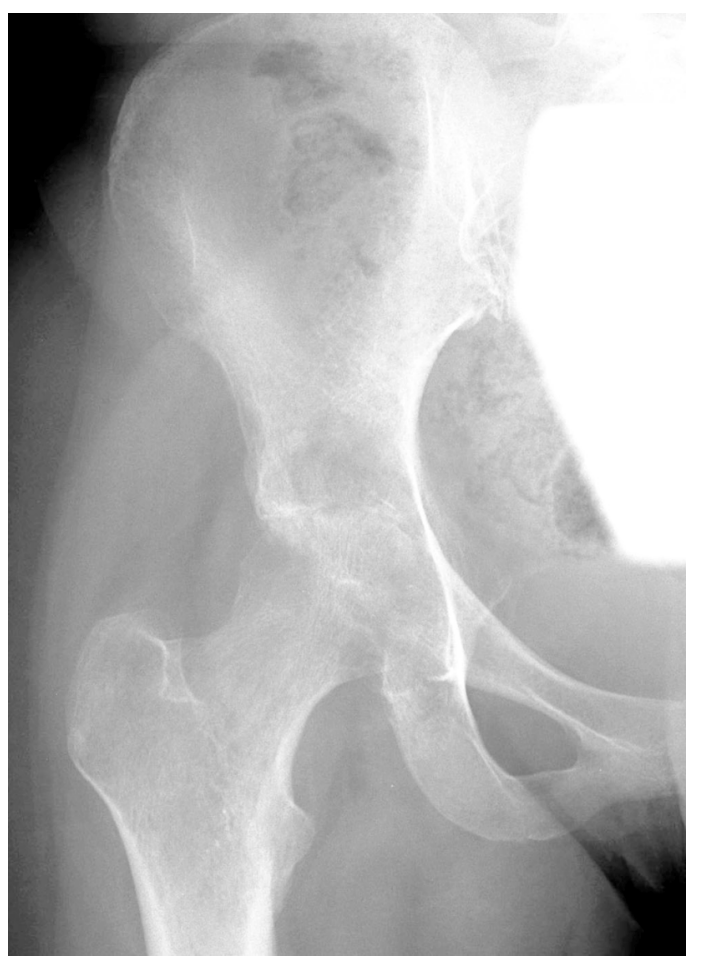

Fig. (2). X-ray of the right pelvis 3 months after diagnosis with increasing patchy transparence in the femoral head and neck as well as in the greater trochanter and acetabulum with clear progressive joint space narrowing.

After seven months the microbiological examination of gastric secretion and sputum analysis did not detect open lung tuberculosis, so the oral therapy was reduced to double combination with Isoniazid and Rifampicin. In contrast to Xray and MRI results with progressive destruction, the ROM of the right hip joint improved. The patient underwent reconstructive surgery by extirpation of the granuloma from the acetabulum and augmentation with autologous spongiosaplasty from the ipsilateral iliac crest. Microbiological and microscopic examination of intraoperative swabs and surface biopsies did not detect M. tbc or other pathological results.

Six weeks after surgery, continuing physiotherapy and oral double combination, the patient was almost free of pain and ROM was improved. X-ray of the right pelvis revealed an unchanged result compared to the examination after operation without any progression of destruction and regular positioned autologous spongiosaplasty without dislocation. The physical load for the right leg was gradually increased and ROM was continuously improved.

Ten months after starting oral therapy, a CT of the thorax detected scars without nodular pathologic infiltrates or pleural effusion in both apical parts of the lungs.

The ROM of the hip joint in spite of the radiological findings with progressive sclerosis in the acetabulum and joint space narrowing (Fig. 4) was satisfactory measured with $125^{\circ}$ for flexion, $35^{\circ}$ for abduction, $20^{\circ}$ for adduction, $5^{\circ}$ for internal rotation and $30^{\circ}$ for external rotation. The 
patient was able to walk without crutches suffering only minor pain.

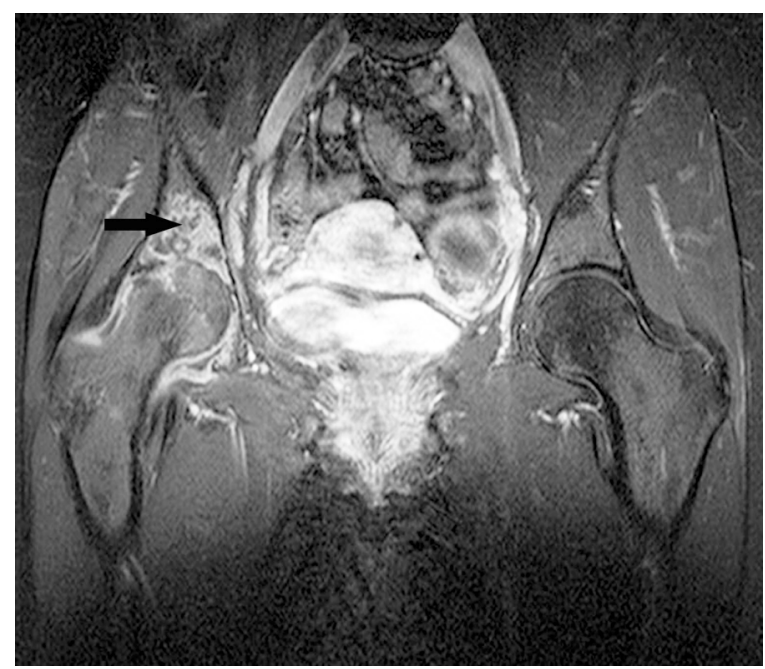

Fig. (3). MRI 5 months after starting the oral therapy showing an active arthritis with progressive destruction of the right hip joint and liquid retention in the right acetabulum as a sign of tuberculous osteomyelitis (see black arrow).

\section{DISCUSSION}

Tuberculosis belongs to the most important infectious diseases worldwide. Although its prevalence in industrial countries is decreasing, it still is not eradicated. According to previous analyses about $10 \%$ of extrapulmonary manifestations refer to joints and bone, mainly to the spinal column and the hip joint. Thus tuberculosis is considered to be a significant disease in discovering diagnosis of uncertain complaints in joints and bones. The early diagnosis could be difficult as the primary clinical symptoms and radiological findings at an early stage are often non-specific [5].

In our case the initial radiographic findings, MRI and standard blood samples were unspecific. Further clinical examination with detailed reconstruction of patient's history (extirpation of suspicious lymph node two years before) as well as her profession as a veterinarian led to the suspicion of tuberculosis, which was confirmed by microbiological cultures of the aspirate and a Quantiferon-Test [7].

In case of TBC prior pulmonary infection causes the affection of the joint by haematogenous spread so a consequently performed $\mathrm{CT}$ of the chest revealed pulmonary infiltrates as well as microbiological examinations of the gastric secretion and sputum analysis showed open tuberculosis. Consequently, the patient must have had an exposure to M. tbc, which retrospectively could have been most probably due to contact with an infected animal [8-10]. However, there are several recommendations for the period of treatment and combination of antituberculotic medication before an operative treatment $[11,12]$ to reduce potential risk of reactivation of infection.

Furthermore different surgical techniques as arthrotomy with debridement, arthrodesis, Girdlestone resection arthroplasty or total hip arthroplasty (THA) [13-18] have been performed in cases of extensive disease [5, 19]. Although Girdlestone resection arthroplasty is not difficult to perform and in most of the cases clinical results are satisfactory [20-22], the major disadvantage of this technique is loss of function due to reduced hip offset. Arthrodesis as an alternative represents to reduce pain and in the meantime treats the infection successfully. Besides loosing ROM of the hip joint, there are often secondary degenerative changes especially of lumbar spine in the longer term. Performing a THA should be mentioned as a demanding alternative, which is often practiced in combination with oral antituberculotic medication. Local exacerbation is observed in cases with insufficient systemic treatment, whereas in most of the cases the results referring to ROM and pain-reduction are satisfactory.

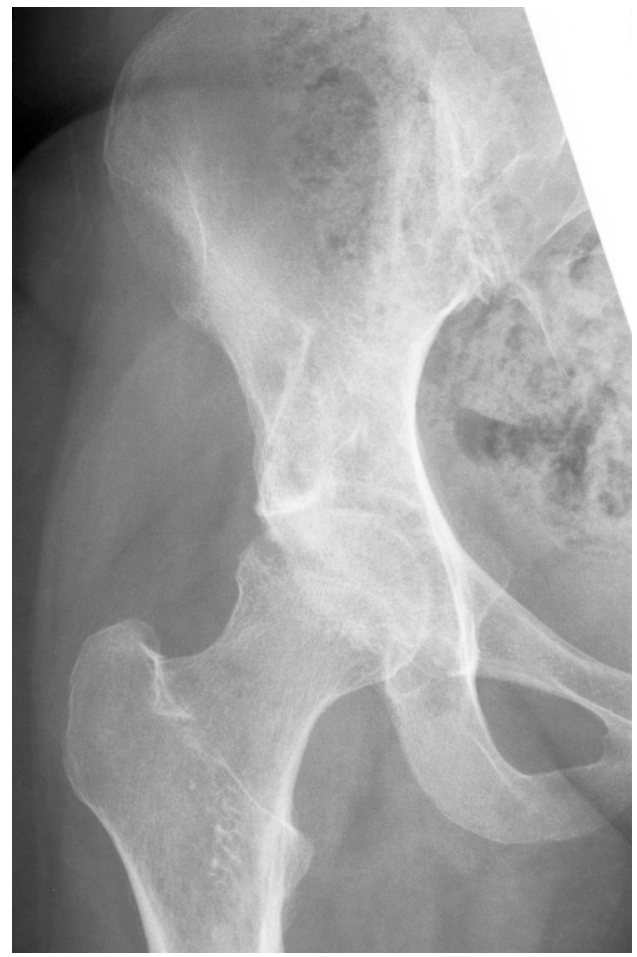

Fig. (4). X-ray oft the right pelvis 1 year after diagnosis showing a progressive sclerosis of the acetabulum with decreasing joint space narrowing.

In our case the patient underwent reconstructive surgery by extirpation of the granuloma in the acetabulum and augmentation with autologous spongiosaplasty after oral antituberculotic treatment.

The reason for our decision to choose a reconstructive bone-saving surgical technique was influenced by different factors.

It is reported that extensive operative interventions as THA for TBC continue to be a controversial issue due to the potential risk of reactivation of infection [5]. However, the period of time after beginning oral antituberculotic treatment and performing an arthroplasty is discussed [23, 24].

An argument against Girdlestone resection or arthrodesis was the improvement of ROM as well as the reduction of pain supported by a regular performed physiotherapy during the period before operative intervention.

Furthermore the patient was young and had the compliance for a distinguished rehabilitation. Moreover she was not convinced of a more extensive surgical intervention 
and agreed with the operation as an attempt to improve her situation.

\section{CONCLUSION}

In conclusion this case demonstrates, that tuberculosis should still be considered as a possibility of a specific origin of joint infection in finding the diagnosis of uncertain complaints of joints without initial radiographic abnormabilities, even in otherwise healthy and young patients without obvious risk factors.

It shows the importance of an extended individual examination with a detailed evaluation of patient's medical history as well as conventional radiography, CT, MRI and microbiological analysis.

Considering the different kinds of surgical techniques and preoperative oral antituberculotic therapies, the decision for a concept of treatment is influenced by several factors as the individual situation of the patient, the compliance, ROM and the initial destruction of the joint. Furthermore the preoperative response to oral antituberculotic therapy as well as persistent histological evidence of active disease are important aspects for choosing the suitable surgical technique for operative treatment.

Finally it should be emphasized, that a decision for performing a less extensive bone-saving operative intervention for the treatment of $\mathrm{TBC}$ as a first step with a minimal surgical expenditure for patient and surgeon in a suitable case does not exclude further application of more extensive surgical treatment, thus the above mentioned surgical techniques could be still performed in case of failure.

\section{AUTHORS' CONTRIBUTIONS}

HK analyzed and interpreted all patient data and was major contributor in writing the manuscript. IS was involved in revising the manuscript critically for important intellectual content. All authors read and approved the final manuscript.

\section{CONFLICT OF INTEREST}

The authors confirm that this article content has no conflicts of interest.

\section{ACKNOWLEDGEMENT}

Declared none.

\section{REFERENCES}

[1] Schweisfurth H, Link K. Clinical studies of the incidence of pulmonary and extrapulmonary tuberculosis. Versicherungsmedizin 1995; 47(6): 207-12.

[2] Hauer B, Brodhun B, Altmann D, Sagebiel D, Haas W, Loddenkemper R. Tuberculosis in Germany in 2001 and 2002. Pneumologie 2005; 59(4): 264-9.
[3] Leowski J, Miller M. Tuberculosis and AIDS: European and worldwide perspectives. Soz Praventivmed 1992; 37(5): 199-206.

[4] Forssbohm M, Zwahlen M, Loddenkemper R, Rieder HL. Demographic characteristics of patients with extrapulmonary tuberculosis in Germany. Eur Respir J 2008; 31(1): 99-105.

[5] Caparros AB, Sousa M, Ribera Zabalbeascoa J, Uceda Carrascosa P, Moya Corral F. Total hip arthroplasty for tuberculous coxitis. Int Orthop 1999; 23(6): 348-50

[6] Meziane A, Messaoudi H, Bahechar N, Ouadfel J, Beldjelti S. Tuberculosis of the hip: diagnostic problems. Apropos of 44 cases. J Chir (Paris) 1987; 124(11): 612-4.

[7] Ak O, Dabak G, Ozer S, Saygi A, Dabak R. The evaluation of the Quantiferon-TB Gold test in pulmonary and extrapulmonary tuberculosis. J Jpn J Infect Dis 2009; 62(2): 149-51.

[8] Fanning A, Edwards S, Hauer G. Mycobacterium bovis infection in humans exposed to elk in Alberta. Can Dis Wkly Rep 1991; 17(44): 239-40.

[9] Fritsche A, Engel R, Buhl D, Zellweger JP. Mycobacterium bovis tuberculosis: from animal to man and back. Int J Tuberc Lung Dis 2004; 8(7): 903-4.

[10] Veterinarians NAoSPH; Centers for Disease Control and Prevention (CDC); Council of State and Territorial Epidemiologists; American Veterinary Medical Association: Compendium of measures to prevent disease associated with animals in public settings 2007. MMWR Recomm Rep 2007; 56 (RR-5): 1-14.

[11] Dutt AK, Moers D, Stead WW. Short-course chemotherapy for extrapulmonary tuberculosis. Nine years' experience. Ann Intern Med 1986; 104(1): 7-12.

[12] Watts HG, Lifeso RM. Tuberculosis of bones and joints. J Bone Joint Surg Am 1996; 78(2): 288-98.

[13] Eskola A, Santavirta S, Konttinen YT, Tallroth K, Hoikka V, Lindholm ST. Cementless total replacement for old tuberculosis of the hip. J Bone Joint Surg Br 1988; 70(4): 603-6.

[14] Hardinge K, Cleary J, Charnley J. Low-friction arthroplasty for healed septic and tuberculous arthritis. J Bone Joint Surg Br 1979; 61-B(2): 144-7.

[15] Kim YH, Han DY, Park BM. Total hip arthroplasty for tuberculous coxarthrosis. J Bone Joint Surg Am 1987; 69(5): 718-27.

[16] Kim YY, Ko CU, Ahn JY, Yoon YS, Kwak BM. Charnley low friction arthroplasty in tuberculosis of the hip. An eight to 13-year follow-up. J Bone Joint Surg Br 1988; 70(5): 756-60.

[17] Ozturkmen Y, Karamehmetoglu M, Leblebici C, Gokce A, Caniklioglu M. Cementless total hip arthroplasty for the management of tuberculosis coxitis. Arch Orthop Trauma Surg 2010; 130(2): 197-203.

[18] Barfod K, Broeng L, Meyer CN. Tuberculous coxitis in the hips 55 years after primary tuberculosis. Ugeskr Laeg 2011; 173(23): 16534.

[19] Netval M, Tawa N, Chocholac D. Total hip replacement after tuberculous coxitis. Twenty-seven-year experience (1980-2007). Acta Chir Orthop Traumatol Cech 2008; 75(6): 446-50.

[20] Haw CS, Gray DH. Excision arthroplasty of the hip. J Bone Joint Surg Br 1976; 58(1): 44-7.

[21] Sharma S, Gopalakrishnan L, Yadav SS. Girdlestone arthroplasty. Int Surg 1982; 67(4 Suppl): 547-50.

[22] Tuli SM, Mukherjee SK. Excision arthroplasty for tuberculous and pyogenic arthritis of the hip. J Bone Joint Surg Br 1981; 63-B(1): 29-32.

[23] Fink B, Casser HR, Zilkens KW, Ruther W. Reactivation of a tuberculous coxitis due to loosening of a total hip endoprosthesis. Arch Orthop Trauma Surg 1995; 114(5): 298-301.

[24] Johnson R, Barnes KL, Owen R. Reactivation of tuberculosis after total hip replacement. J Bone Joint Surg Br 1979; 61-B(2): 148-50. 